\title{
Role of Multidetector CT Imaging in the Risk Stratification of Gastrointestinal Stromal Tumors (GISTs)-A Retrospective Analysis
}

\author{
Geena Benjamin ${ }^{1}$ Thara Pratap ${ }^{2}$ Mangalanandan Sreenivasan ${ }^{1}$ Dhanya Jacob ${ }^{2}$ Agnes Thomas ${ }^{3}$ \\ Bala Sankar ${ }^{1}$ Amith Itty ${ }^{1}$
}

1Department of Radiology, Pushpagiri Institute of Medical Sciences
\& Research Centre, Thiruvalla, Kerala, India
2Department of Radiology, VPS Lakeshore Hospital, Kochi, Kerala,
India
${ }^{3}$ Department of Radiology, Mar Sleeva Medicity, Palai, Kerala, India

J Gastrointestinal Abdominal Radiol ISGAR 2021;4:14-23.

\begin{abstract}
Address for correspondence Geena Benjamin, MBBS, DMRD, DNB, FRCR, Department of Radiology, Pushpagiri Institute of Medical Sciences \& Research Centre, Thiruvalla 689103, Kerala, India (e-mail: geenabenjamin@gmail.com).
\end{abstract}

\begin{abstract}
Keywords

- GIST

- MDCT

- risk stratification

Background Gastrointestinal stromal tumors (GISTs) are the most common gastrointestinal mesenchymal neoplasms which can arise from any part of the gastrointestinal tract (GIT) or an extraintestinal location. Size and the organ of origin are the major imaging inputs expected from the radiologist. However, it is worthwhile to find out which imaging characteristics on MDCT correlate with risk stratification. This knowledge would help the clinician in treatment planning and prognostication. The aim of this retrospective study is to evaluate the various MDCT imaging characteristics of GISTs and find out which parameters have significant association with risk and subsequent development of metastasis on follow-up whenever it was possible.

Materials and Methods This is a retrospective study conducted on 45 histopathologically proven cases of GIST from two institutions by searching from the digital archives. The following imaging parameters were analyzed: maximum size in any plane, organ of origin, shape (round, ovoid or irregular), margin (well-defined or ill-defined), surface (smooth or lobulated), percentage of necrosis, growth pattern, enhancement characteristics-both intensity (mild, moderate or significant) and pattern (homogenous vs. heterogenous), calcification, infiltration into adjacent organs, and presence of metastasis at presentation or on follow-up.

Results CT morphological parameters of significance in risk stratification as per our study include tumor necrosis, predominant cystic change, irregular and lobulated shape/surface characteristics, and adjacent organ infiltration.

The parameters which were associated with development of metastasis were size $>5 \mathrm{~cm}$, necrosis $>30 \%$, and the presence of adjacent organ infiltration.

Conclusion The radiologist has an important role in ascertaining the size of tumor as well as the organ of origin accurately to guide the clinician in risk calculation and subsequent prognostication. In addition, certain CT characteristics mentioned above, namely, tumor size, significant necrosis/cystic changes, irregular/lobulated contour, and invasion of adjacent organs, help in risk stratification and in predicting metastasis/ poor prognosis.
\end{abstract}

published online

January 10, 2021
DOI https://doi.org/

$10.1055 / \mathrm{s}-0040-1716789$ ISSN 2581-9933. (c) 2021. Indian Society of Gastrointestinal and Abdominal Radiology.

This is an open access article published by Thieme under the terms of the Creative Commons Attribution-NonDerivative-NonCommercial-License, permitting copying and reproduction so long as the original work is given appropriate credit. Contents may not be used for commercial purposes, or adapted, remixed, transformed or built upon. (https:// creativecommons.org/licenses/by-nc-nd/4.0/)

Thieme Medical and Scientific Publishers Pvt. Ltd. A-12, 2nd Floor, Sector 2, Noida-201301 UP, India 


\section{Introduction}

Gastrointestinal stromal tumors (GISTs) were considered as smooth muscle tumors along with leiomyoma, leiomyoblastoma, and leiomyosarcoma before the 1980s. Later with advancements in immunohistochemistry, they were reclassified as a specific group of mesenchymal tumors by Mazur and Clark in 1983, based on their origin from interstitial cells of Cajal. ${ }^{1}$ GISTs are the most common mesenchymal neoplasms of the gastrointestinal tract (GIT). The interstitial cells of Cajal which are located in the myenteric plexus express a tyrosine kinase growth factor receptor named c-kit protein (CD-117), a specific immunohistochemical (IHC) marker which is not seen in smooth muscle and other mesenchymal tumors of GIT. This marker enabled targeted therapy using Imatinib and other tyrosine kinase inhibitors. ${ }^{2}$

GISTs can occur anywhere along the GIT, from esophagus to rectum and less commonly in extragastrointestinal locations in the abdominal cavity. Stomach is the most common site of occurrence ( - Fig. 1) of GIST in GIT (60-70\%), followed by small intestine (25-35\%) (-Fig. 2) and then colon-rectum and appendix (together 5\%), with esophagus being the

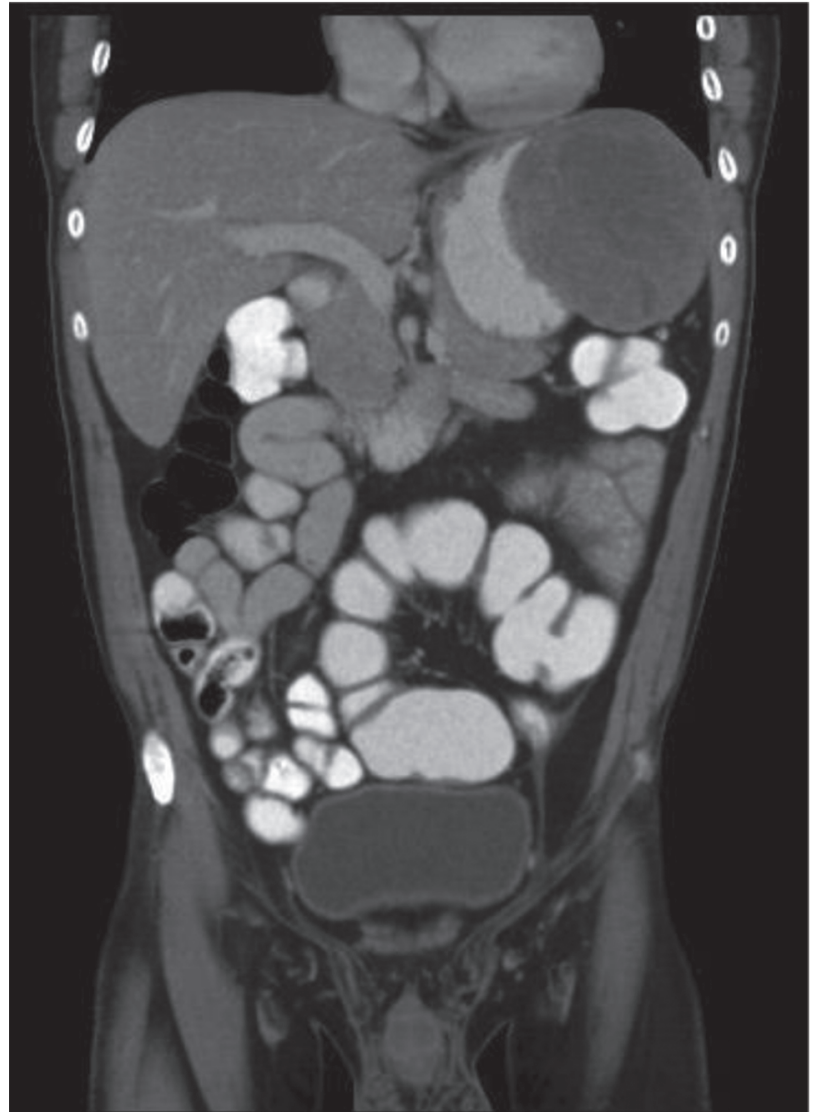

Fig. 1 Coronal contrast-enhanced CT image showing moderate-sized exophytic gastrointestinal stromal tumor (GIST) arising from gastric fundus. High risk. Mitotic index (MI): > 5/50 HPF. Size: $10.3 \mathrm{~cm}$. least common site $(<1 \%)$. In the abdominal cavity, GIST can also arise from omentum, mesentery, and retroperitoneal compartment (less than $5 \%$ of all GISTs). ${ }^{3}$

Depending on the location and size of the GIST, clinical presentation can vary from asymptomatic and incidentally detected lesions to masses, which can cause abdominal pain, bleeding into bowel or abdominal cavity, anemia, dyspepsia, nausea or vomiting, symptoms due to mass effect or, rarely, bowel obstruction. ${ }^{4}$ Only one of the cases presented with small bowel obstruction in our study (-Fig. 3 A, B). Majority of GISTs are benign lesions with 20 to $30 \%$ having malignant potential, which can be identified based on certain imaging and pathological findings.,

Imaging workup for GIST includes endoscopic ultrasonography, CT, MRI and functional imaging with positron emission tomography (PET)-CT. Contrast-enhanced CT (CECT) is the first-line imaging modality in suspected GISTs.

For patients with comorbidities who are high-risk candidates for surgery and for those who refuse surgery, it will be beneficial if imaging morphology could help to a certain extent in risk stratification of GIST. Imaging-guided biopsy could rarely cause tumor dissemination. ${ }^{5}$ It is also a known fact that biopsy specimens are likely to give inaccurate mitotic count due to the small sample size and sampling error, especially in case of a markedly heterogeneous tumor.

Many patients who undergo neoadjuvant therapy with tyrosine kinase inhibitors, especially those with large primary tumors, may face limitation in the mitotic index assessment in the final resected specimen due to therapy-induced tumor changes. It is this knowledge that sparks an interest in assessing the role of tumor imaging morphology in risk stratification and the likelihood of development of metastasis or disease progression. ${ }^{6}$

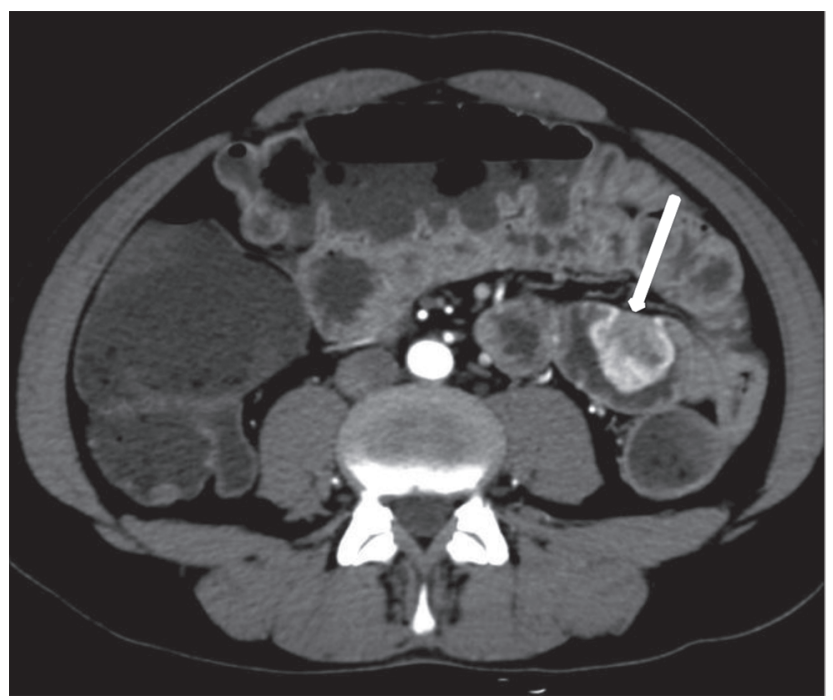

Fig. 2 Axial contrast-enhanced CT image showing intensely enhancing completely endophytic jejunal gastrointestinal stromal tumor (GIST). Low risk. Mitotic index (MI) <5/50 HPF. Size: $3 \mathrm{~cm}$. 

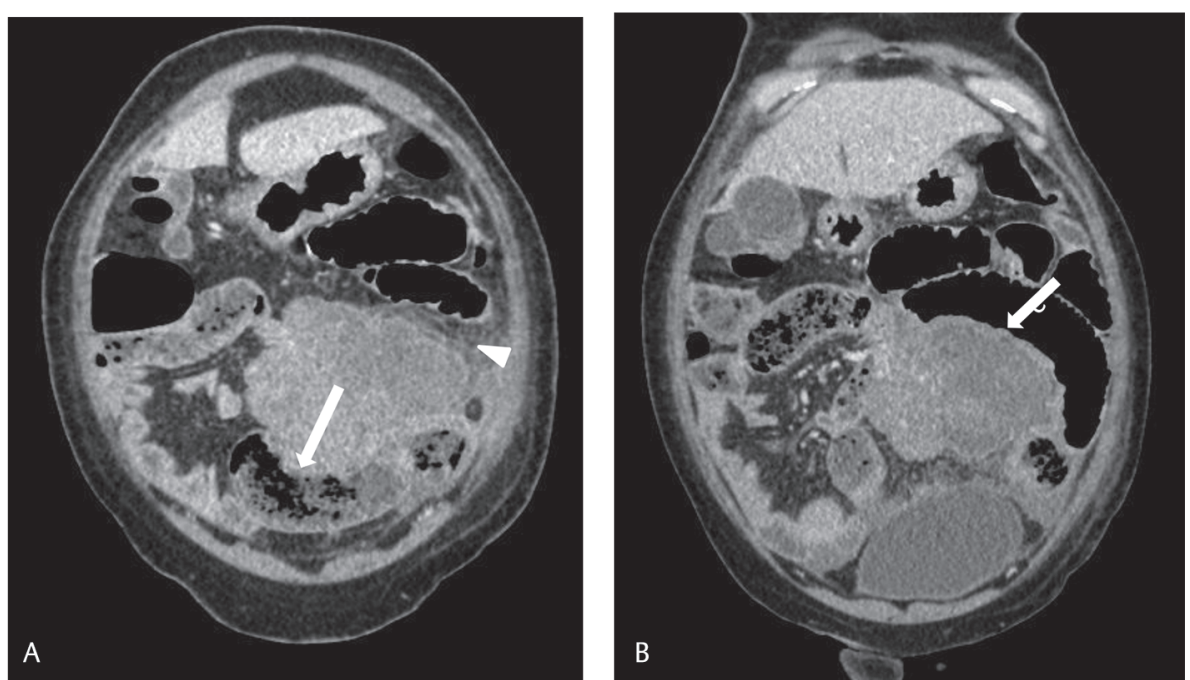

Fig. 3 (A, B) Coronal contrast-enhanced CT images of a 44-year-old female with jejunal gastrointestinal stromal tumor (GIST) showing lobulated and irregular contour, invasion into adjacent small bowel loops (white arrows) and omentum (white arrowhead) with coexistent adhesion causing bowel obstruction. High risk. Size: $11.4 \mathrm{~cm}$. Mitotic index (MI) >5/50 HPF.

The aim of this study is to evaluate the imaging features of GISTs on CECT and assess its role in risk stratification in a selected South Indian population.

\section{Materials and Methods}

\section{Study Population}

This is a retrospective study conducted by consecutive sampling of all patients registered in the oncology departments of the participating institutions in the 6-year period from January 2014 to December 2019, who have undergone CECT and who were subsequently (post-surgical resection) proven to have GISTs based on histopathology and IHC (CD 117/DOG1 positivity). Whenever feasible, the cases were followed-up for a period ranging from 6 to 60 months to look for development of metastasis and correlate the same with mitotic index (MI) and risk stratification. Out of the 45 cases included in this study, only 33 cases could be followed-up.

Data was retrieved from electronic radiology database. A total of 45 patients ( 26 males and 19 females) met the inclusion criteria and were included in the study after excluding patients who did not have pretreatment (prior to surgery or systemic therapy) CT images available for evaluations. We have included gastric, small and large bowel GISTs in our study, all of whom underwent multidetector (MD) CECT prior to surgery or neoadjuvant therapy. We did not have any case of esophageal or extragastrointestinal GIST.

\section{Imaging Technique}

Patients were kept fasting for a minimum period of 6 hours before the abdominal and pelvic CT examination and were made to drink 1000 to $1200 \mathrm{~mL}$ of gastrograffin or mannitol prior to the CT examination. Whenever indicated per rectal contrast was also administered. Patients were in supine position and scanned from domes of diaphragm to pubic symphysis, using 64-slice MDCT GE Lightspeed or 128 slice MDCT, OPTIMA 660 from GE healthcare,
Milwaukee, WI, USA. Following the unenhanced CT, 80 to $120 \mathrm{~mL}$ (1.5 mL/kg body weight) of Iohexol (350 mg iodine/mL; Omnipaque, GE Healthcare, Shanghai, China) or Iopromide (Ultravist, Bayer Zydus Pharma Ltd, NJ, USA) was administered intravenously at a flow rate of $4 \mathrm{~mL} / \mathrm{s}$ using a dual head pressure injector (Medrad Stellant CT Injector System, Indianola, PA, US). CECT images were obtained in late arterial phase (35 seconds) and hepatic venous phase (70 seconds). CT parameters were as follows: $120 \mathrm{kVp}$, automatic tube current modulation, $5 \mathrm{~mm}$ slice thickness with $0.625 \mathrm{~mm}$ reconstruction, 35 to $50 \mathrm{~cm}$ field of view and $512 \times 512$ matrix. Raw data was reconstructed in coronal and sagittal planes.

MRI with contrast was done as a correlative study in few patients $(n=4)$, especially when CT showed very large or predominantly cystic tumor.

\section{Image Analysis}

Three abdominal radiologists (with more than 10 years of experience) reviewed the images in consensus. The images were reviewed on picture archiving and communication system (PACS).

The following imaging parameters were analyzed: maximum size in any plane, organ of origin, shape (round, ovoid or irregular), margin (well-defined or ill-defined), surface (smooth or lobulated), percentage of necrosis, growth pattern, enhancement characteristics-both intensity (mild, moderate or significant) and pattern (homogenous vs. heterogenous), calcification, infiltration into adjacent organs, and presence of metastasis at presentation or on follow-up.

The lesions were classified as those with size less than $5 \mathrm{~cm}$ (small) or more than $5 \mathrm{~cm}$ (large). Lesions arising from stomach, small bowel, large bowel, and rectum were separately classified. The percentage of necrosis in the tumor was categorized as less than 30\%, 30 to 50\%, more than 50\% and predominantly cystic (when more than $50 \%$ of the tumor showed well defined cystic change). 
Growth patterns were classified as endophytic, exophytic, or mixed. Endoluminal growth was noted when a tumor was attached to the gastric or bowel wall and was completely within the lumen without bulging into the extraluminal space ( - Fig. 4A, B). Exophytic growth was stated when a mass was confined to the extraluminal space without bulging into the gastric or bowel lumen. When tumors had both endo and exophytic components they were said to have mixed growth pattern ( - Fig. 5A-C).

To determine the tumor enhancement intensity, CT attenuation values of the lesion were measured in Hounsfield units (HU). Mild enhancement was when the $\mathrm{HU}$ was less than 60 , moderate in the range of 60 to $100 \mathrm{HU}$, and significant being more than $100 \mathrm{HU}$.

The histopathological correlation has been done with cell type (spindle cell, epithelioid cell or mixed), MI (less than or more than 5 mitosis per 50 high power field [HPF]), and IHC characteristics especially CD-117 and DOG1.

Risk stratification was done in our study using Miettinen and Lasota's risk stratification criteria (2006). This risk stratification system is based on three parameters, that is, MI, organ of origin and largest diameter of the tumor. Based on this, when the mitotic count is $>5 / 50 \mathrm{HPF}$, all GISTs $>5 \mathrm{~cm}$ in size, irrespective of site of GIT origin, fall into high-risk category.
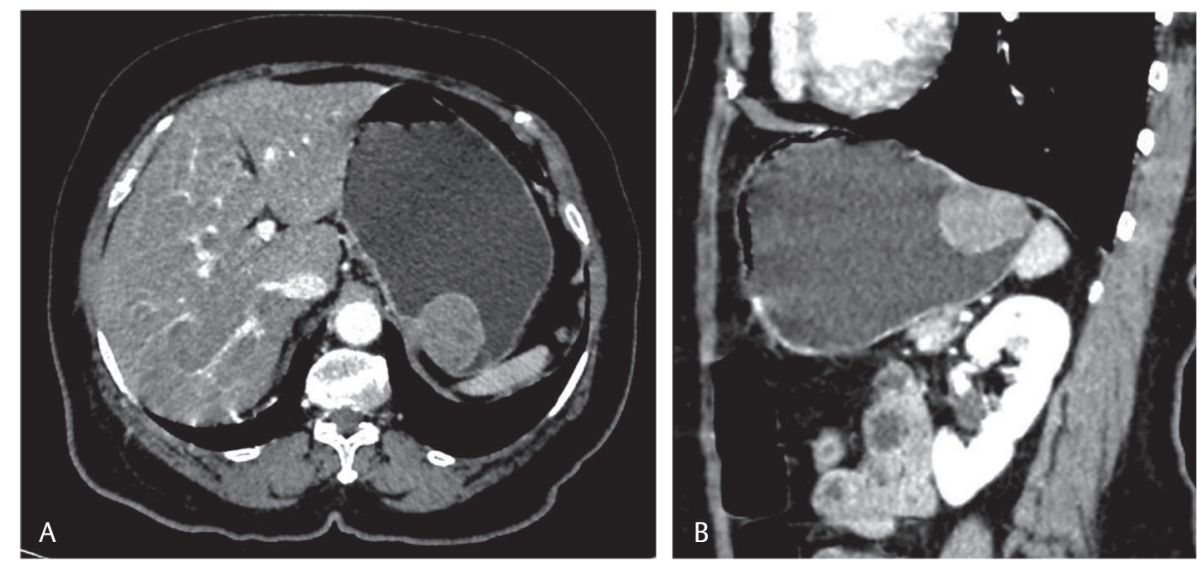

Fig. 4 (A) Axial and (B) sagittal reformatted contrast-enhanced CT images of 64-year-old female with totally endophytic gastric gastrointestinal stromal tumor (GIST). Size $3.7 \mathrm{~cm}$. Mitotic index (MI) < 5/50 HPF. Low risk.
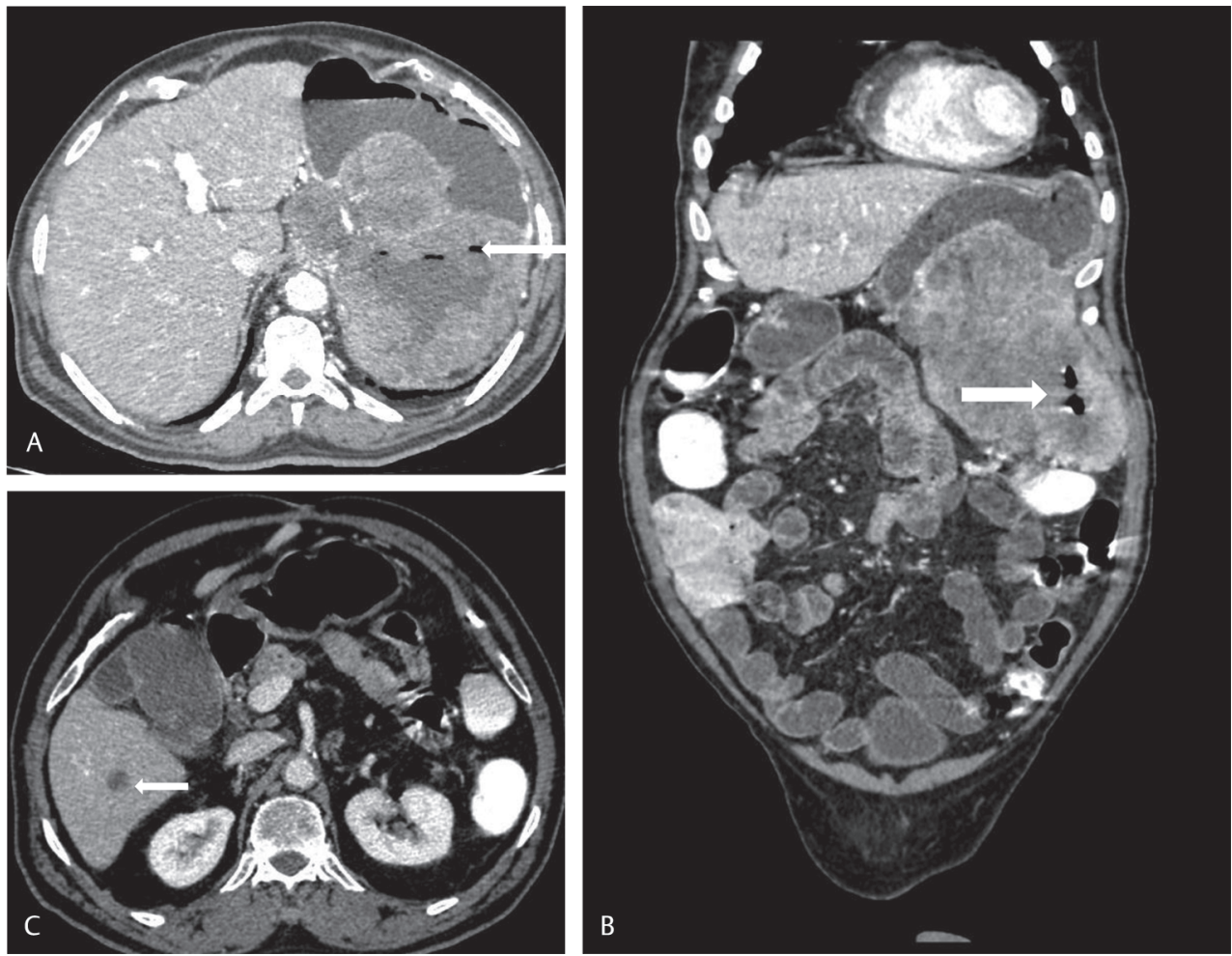

Fig. 5 (A) Axial and (B) coronal reformatted contrast-enhanced CT images of a large gastric gastrointestinal stromal tumor (GIST) showing endoexophytic growth pattern and associated fistulous communication with gastric lumen. White arrows point to air pockets within the central necrotic portion of tumor. Tumor had > 30\% necrosis. High risk. Mitotic index (MI) >5/50 HPF. Size $16.2 \mathrm{~cm}$. (C) Axial CECT taken 6 months after surgery shows cystic metastasis within central necrotic portion of tumor which confirms fistulous communication in right lobe of liver (white arrow). 
When the size is between 2 and $5 \mathrm{~cm}$, gastric GISTs fall into intermediate risk, whereas small and large bowel tumors stay in high-risk category. When the size is $<2 \mathrm{~cm}$, even with mitotic count $>5 / 50 \mathrm{HPF}$, gastric GISTs have only negligible risk, whereas small bowel and colorectal GISTs fall in the highrisk category. When the mitotic count is $<5 / 50 \mathrm{HPF}$, gastric GISTs of size $>10 \mathrm{~cm}$ fall into intermediate risk, whereas small bowel and colorectal tumors $>5 \mathrm{~cm}$ fall into intermediate or high-risk category.

\section{Statistical Analysis}

Categorical and quantitative variables were expressed as frequency (percentage) and mean $\pm S D$, respectively. Diagnostic statistics such as sensitivity, specificity, positive predictive value (PPV), negative predictive value (NPV), and accuracy have been calculated to assess predictive accuracy of CT imaging morphological parameters of the tumor in detecting risk and mitotic index. Kappa statistics was performed to find the agreement of results of CT imaging parameters with risk and MI. Chi-square test, odds ratio with $95 \% \mathrm{CI}$ was used to find association of risk and MI with selected CT imaging parameters. $p<0.05$ was considered as the threshold for statistical significance. Statistical analyses were performed by using a statistical software package SPSS, version 20.0.

\section{Results}

Each of the 12 imaging parameters were analyzed. Note was also made of the age and sex distribution of the study population. In our study, the patients were in the age ranging from 35 to 82 years. Of this, $51.1 \%$ of cases belonged to the 6 th and 7 th decades. The average age was 61 years with SD of 12.31 . As much as $57.8 \%$ of our cases were males and $42.2 \%$ of the cases were females.

- Table 1 shows the percentage distribution of the sample according to the various $\mathrm{CT}$ imaging characteristics. As many as 29 of the 45 cases (64.4\%) showed size more than $5 \mathrm{~cm}$. All the patients who showed homogenous tumor enhancement had size $<5 \mathrm{~cm}$. Reports were analyzed separately with percentage of tumor necrosis cutoff at $50 \%$ and also at $30 \%$. Those with $>50 \%$ necrosis $(n=9)$ or predominantly cystic change $(n=7)$ together constituted $35.6 \%(n=16)$. Those with necrosis more than $30 \%$ combined with tumors with cystic change constituted $51 \%(n=23)$. Adjacent organ infiltration was seen in $28.8 \%$ of the cases ( $\mathbf{- F i g . 6} \mathbf{~ A - D}$ ). As much as $66.6 \%$ cases had either irregular or lobulated shape/surface $(n=30)$. As much as $87 \%$ cases were of spindle cell type on histopathologic evaluation. Out of 45 patients, 43 showed CD 117 positivity. DOG-1 positivity was documented in the two patients who were CD 117 negative. As much as 42.2\% of cases $(n=19)$ had mitotic rate of $>5 / 50$ HFP and $57.8 \%$ $(n=26)$ had mitotic rate of $<5 / 50 \mathrm{HPF}$.

Most of our patients (57.8\%) were in the high-risk category, $15.6 \%$ were in the moderate/intermediate risk group, followed by $13.3 \%$ in the very low, $11.1 \%$ in the low-risk group and $2.2 \%$ had zero risk. In our study, cases with no risk, very low
Table 1 CT morphological characteristics of patients selected for study.

\begin{tabular}{|c|c|c|c|}
\hline \multicolumn{2}{|c|}{ CT characteristics } & \multirow{2}{*}{$\begin{array}{l}\text { Count } \\
16\end{array}$} & \multirow{2}{*}{$\begin{array}{l}\text { Percentage } \\
35.6\end{array}$} \\
\hline Size & $<5$ & & \\
\hline & $5-10$ & 13 & 28.9 \\
\hline & $>10$ & 16 & 35.6 \\
\hline \multirow[t]{3}{*}{ Shape } & Round & 15 & 33.3 \\
\hline & Ovoid & 20 & 44.4 \\
\hline & Irregular & 10 & 22.2 \\
\hline \multirow[t]{2}{*}{ Margins } & Well-defined & 42 & 93.3 \\
\hline & III-defined & 3 & 6.7 \\
\hline \multirow[t]{2}{*}{ Surface } & Smooth & 15 & 33.3 \\
\hline & Lobulated & 30 & 66.7 \\
\hline \multirow[t]{3}{*}{ Shape/surface } & Irregular and lobulated & 10 & 22.2 \\
\hline & Regular and lobulated & 20 & 44.4 \\
\hline & Regular and smooth & 15 & 33.3 \\
\hline \multirow{3}{*}{$\begin{array}{l}\text { Enhancement } \\
\text { intensity }\end{array}$} & Mild & 21 & 46.7 \\
\hline & Moderate & 18 & 40.0 \\
\hline & Significant & 6 & 13.3 \\
\hline \multirow{2}{*}{$\begin{array}{l}\text { Pattern of } \\
\text { Enhancement }\end{array}$} & Heterogeneous & 36 & 80.0 \\
\hline & Homogeneous & 9 & 20.0 \\
\hline \multirow[t]{5}{*}{ Necrosis } & Absent & 11 & 24.4 \\
\hline & Less than $30 \%$ & 11 & 24.4 \\
\hline & $30-50 \%$ & 7 & 15.6 \\
\hline & $>50 \%$ & 9 & 20.0 \\
\hline & Predominantly cystic & 7 & 15.6 \\
\hline \multirow{2}{*}{$\begin{array}{l}\text { Type of } \\
\text { calcification }\end{array}$} & Present & 11 & 24.4 \\
\hline & Absent & 34 & 75.6 \\
\hline \multirow{8}{*}{$\begin{array}{l}\text { Organ of } \\
\text { metastasis }\end{array}$} & $\mathrm{Nil}$ & 35 & 77.8 \\
\hline & Liver & 4 & 8.9 \\
\hline & Lung & 0 & 0.0 \\
\hline & Lymph node & 0 & 0.0 \\
\hline & Bone & 0 & 0.0 \\
\hline & Peritoneum & 3 & 6.7 \\
\hline & Liver, lymph node & 1 & 2.2 \\
\hline & Liver, peritoneum & 2 & 4.4 \\
\hline \multirow[t]{6}{*}{ Organ of origin } & Stomach & 26 & 57.8 \\
\hline & GE junction & 0 & 0.0 \\
\hline & Small bowel & 12 & 26.7 \\
\hline & Large bowel & 2 & 4.4 \\
\hline & Rectum & 5 & 11.1 \\
\hline & Others & 0 & 0.0 \\
\hline \multirow{3}{*}{$\begin{array}{l}\text { Growth } \\
\text { pattern }\end{array}$} & Exophytic & 25 & 55.6 \\
\hline & Endophytic & 18 & 40.0 \\
\hline & Mixed & 2 & 4.4 \\
\hline
\end{tabular}

Abbreviation: GE, gastroesophageal.

risk and low risk were together included as low-risk group, constituting $26.6 \%(n=12)$. Moderate and high-risk cases were together included under high-risk group, constituting $73.4 \%$ $(n=33)$. One gastric GIST showed fistulous communication with lumen ( - Fig. 5 A, B). 

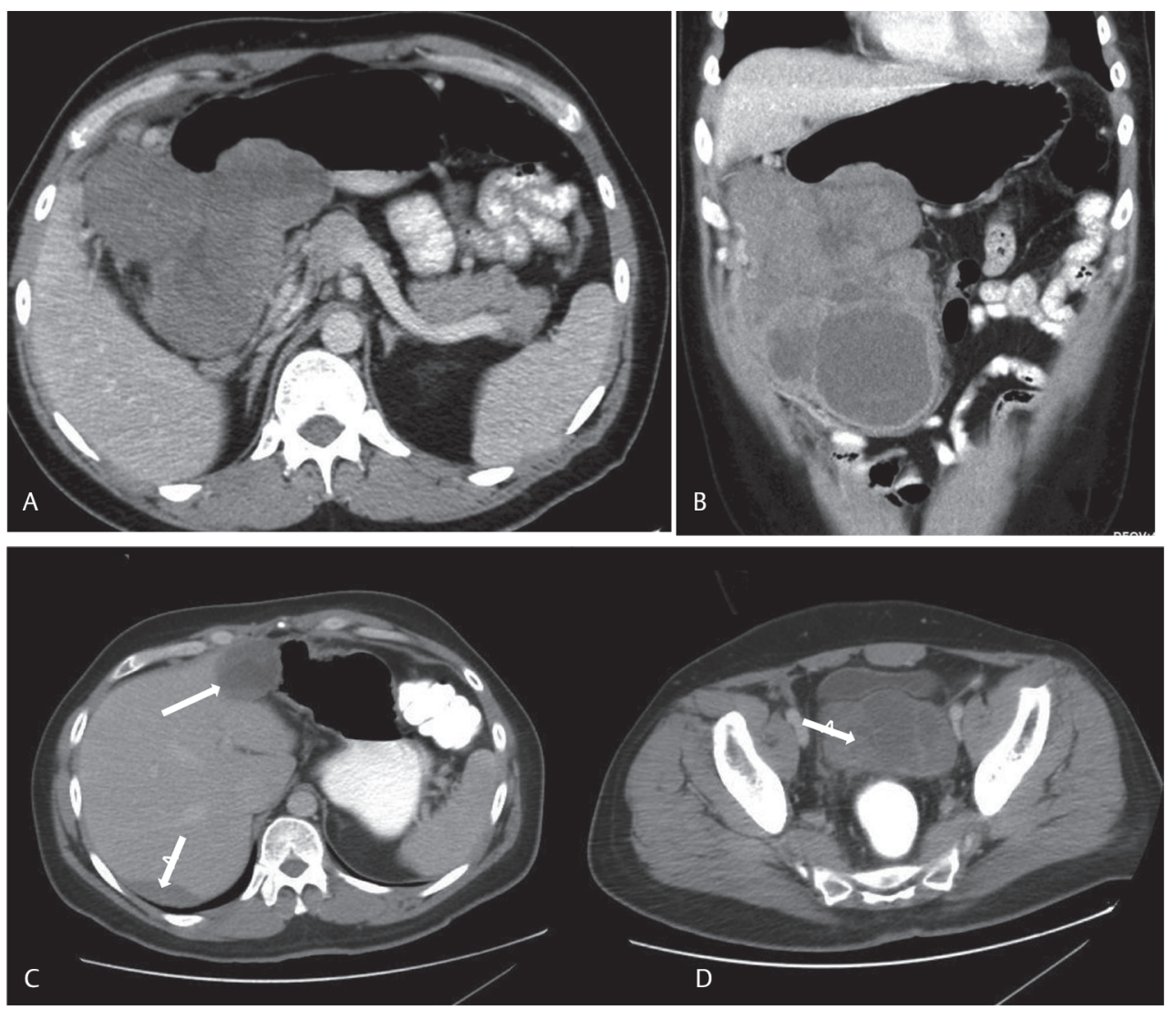

Fig. 6 (A) Axial and (B) coronal contrast-enhanced CT images showing markedly necrotic ascending colonic gastrointestinal stromal tumor (GIST) with lobulated and slightly irregular contour in right hypochondrium and lumbar region, displacing the bowel loops medially and with infiltration into stomach. High risk. Mitotic index (MI) $>5 / 50 \mathrm{HPF}$. Size $15.8 \mathrm{~cm}$. (C) axial upper abdomen and (D) axial pelvic contrast-enhanced CT images of follow-up scan, 1-year postsurgery showing recurrence at the postoperative site near the stomach as well as perihepatic and pelvic deposits (white arrows).

\section{Diagnostic Accuracy of CT Variables in Predicting Risk}

With regard to size of tumor and risk, there was sensitivity and specificity of $81.8 \%$ and $83.3 \%$, respectively, with PPV of $93.1 \%$, accuracy of $82.2 \%$, Kappa 0.59 (moderate agreement) and $p$-value of $<0.01$. The diagnostic accuracy of margins in predicting risk was only 33.3 , with $100 \%$ specificity and PPV, but very low sensitivity. Similarly, intensity of enhancement, enhancement pattern, tumor calcification, and growth pattern failed to show significant agreement in risk stratification.

High percentage of necrosis, irregular or lobulated shape/surface characteristics and adjacent organ infiltration were the imaging parameters which had agreement and significant association with high risk $(p<0.05$;

-Fig. 7 A, B).

There was significant association between percentage of necrosis $>30 \%$ and risk category with $p$-value of $<0.01$, sensitivity $69.7 \%$, specificity and PPV of $100 \%$ each, Kappa $=$ 0.55 and diagnostic accuracy of $77.8 \%$. None of the low risk cases had $>30 \%$ necrosis. Percentage of necrosis showed fair agreement in predicting risk when cutoff was $50 \%$ and moderate agreement when the cutoff was fixed at $30 \%$. All the cases with predominant cystic changes were in the high-risk category.
Tumors with lobulated and irregular shape/surface characteristics showed significant association with risk category with $p$-value of 0.01 . Adjacent organ infiltration showed moderate agreement in risk prediction with Kappa $=0.26$, specificity and PPV of $100 \%$ each, and $p$-value of 0.01 .

GISTs in extragastric location had a greater chance of being high risk when compared with gastric GIST. Only $61.5 \%$ of gastric GISTs were in high-risk category, whereas $83.3 \%$ of small bowel GISTs and $100 \%$ each of rectal and colonic GISTs were in the high-risk category.

\section{Diagnostic Accuracy of CT Variables in Predicting Mitotic Index}

Size of lesion $>5 \mathrm{~cm}$ showed significant association with MI with $p$-value of 0.003 . Irregular/lobulated shape/surface characteristics had association with MI with $p$-value of 0.04 . As far as growth pattern was concerned, $72 \%$ of tumors with exophytic growth pattern had low MI, whereas 55.6\% with endophytic pattern showed high MI with $p$-value of 0.04 . Out of the 13 tumors in our study that showed adjacent organ infiltration, 10 showed high MI (76.9\%), while 23 cases which lacked adjacent infiltration (71.8\%) had low MI, with $p$-value of 0.003 . There was no significant association between percentage of necrosis and $\mathrm{MI}$ in our study 

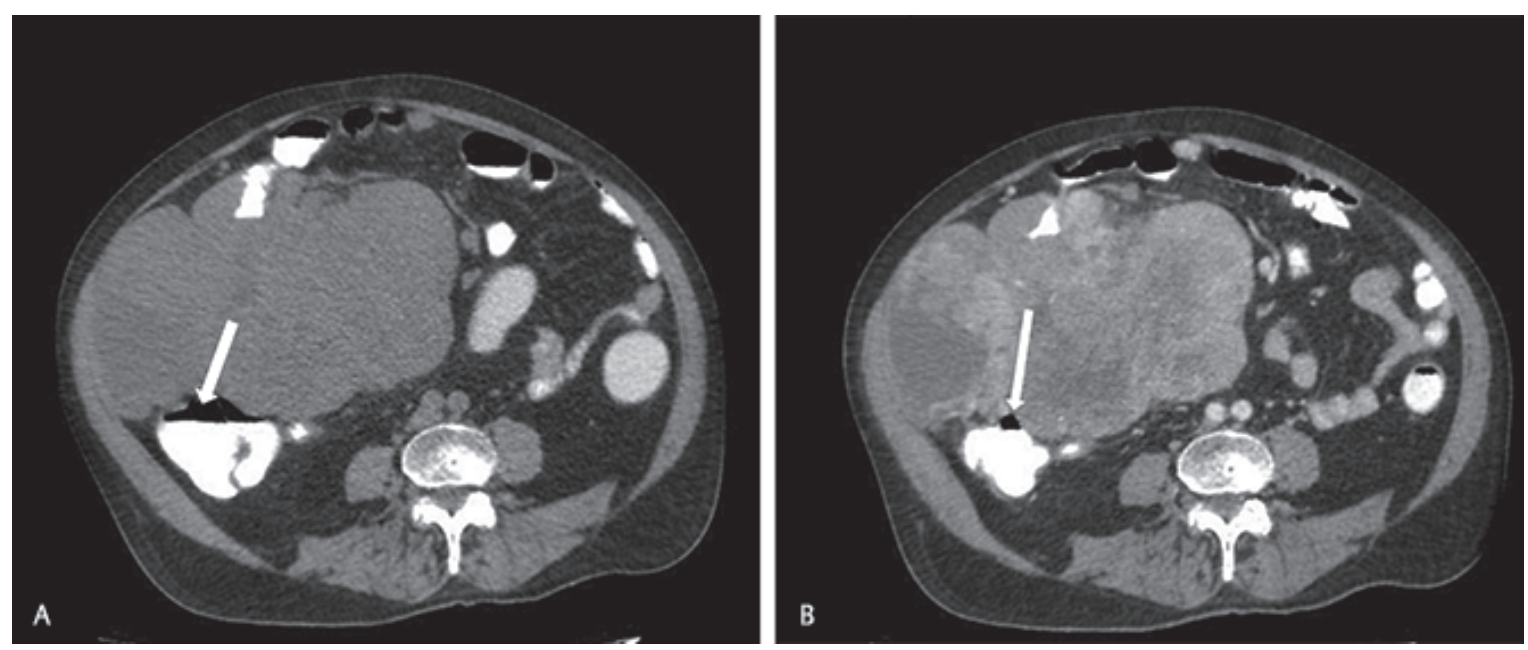

Fig. 7 (A) Plain and (B) contrast-enhanced CT axial images show large exophytic moderately enhancing jejunal gastrointestinal stromal tumor (GIST) with central necrosis and lobulated and irregular contour. There was invasion of adjacent ascending colon (white arrow) High risk. Mitotic index (MI): > 5 / 50 HPF. Size: $21 \mathrm{~cm}$.

( $p$-value $=0.157)$. The remaining CT imaging parameters did not show statistically significant association with MI.

\section{Correlation of CT Characteristics of Those Tumors that Metastasized, with MI and Risk Category}

Among the 33 patients who could be followed up, only 10 developed metastases during the period of observation. Liver was the most common site of metastasis followed by peritoneum/omentum.

As much as $90 \%$ of cases $(n=9)$ that developed metastasis had high MI. Only one small bowel GIST which subsequently metastasized had low MI. Thus, there was significant association between MI and development of metastasis $(p<0.01)$.

It was also found that there was significant association between risk category and occurrence of metastasis at presentation or on follow-up with a $p$-value of 0.01 . The specificity and PPV were $100 \%$ each, but with low sensitivity (30.3\%) and accuracy (48.9\%). None of the low-risk cases developed metastasis, but $50 \%$ of the high-risk cases developed metastasis on follow-up.

- Table 2 shows association of metastasis with selected CT imaging characteristics. The individual CT morphological parameters in tumors with metastasis were analyzed, and it was found that among these, size $>5 \mathrm{~cm}(p<0.01)$, extent of necrosis more than $30 \%(p<0.01)$ and presence of adjacent organ infiltration $(p<0.001)$ showed significant association with metastasis. None of the patients with less than $30 \%$ necrosis developed metastasis in our study.

\section{Discussion}

GISTs are the most common mesenchymal tumors of the GIT and comprise a subset of tumors with marked heterogeneity. GISTs are potentially malignant tumors with nearly 20 to 30\% being malignant. They exhibit a wide range of morphological appearance. GISTs are mostly spindle cell tumors with 20 to $25 \%$ of tumors having epithelioid morphology and some having a combination of spindle and epithelioid cells.
Most GISTs show strong and diffuse expression of KIT (CD117). C-kit mutations are seen in around 80 to $85 \%$ of GIST, with recent studies showing other mutations in GIST like PDGFRA (Platelet derived growth factor receptor $\alpha$ ), BRAF V600E and SDH (succinate dehydrogenase gene) mutations. $^{7}$

A small minority (<5\%) may lack KIT expression. An equally sensitive and specific marker is DOG1 (Discovered on GIST-1), which is a recently described protein expressed in GISTs irrespective of mutation status. This is positive in up to $50 \%$ of KIT negative GISTs.

Although GIST can occur in any age group, it is most frequently seen in those above 50 years, with equal prevalence among both sexes. Most of the GISTs occur sporadically with few cases seen in association with inherited syndromes like neurofibromatosis type I (NF1), Carney triad, CarneyStratakis syndrome and familial GIST. ${ }^{8}$

Although CT is the first-line and most preferred imaging modality in suspected GISTs, MRI is also beneficial in predicting the malignant potential of GIST (like presence of intratumoral large cystic changes and low apparent diffusion coefficient [ADC] values). It may also be helpful in very large GISTs where organ of origin may be difficult to ascertain. Diffusion-weighted imaging is said to have a role in prediction of risk.

PET-CT is mostly concerned with post therapy response evaluation and follow-up of GIST, especially in detection of local/distant recurrence, resulting in significant impact on clinical management. ${ }^{9}$

The goal of this study was to evaluate the MDCT imaging morphology of GISTs and more importantly to analyze its role in risk stratification, which has a huge bearing on the treatment and prognostication.

Majority of our cases were in their 6th and 7th decades, with slight male predominance, and stomach being the most common site, followed by small bowel and colorectal GISTs. With regard to the various CT parameters assessed, majority of tumors had size $>5 \mathrm{~cm}$, well-defined margins, lobulated or 
Table 2 Association of metastasis with selected CT imaging variables

\begin{tabular}{|c|c|c|c|c|c|c|c|c|}
\hline & & \multicolumn{4}{|c|}{ Organ of metastasis } & \multirow[t]{3}{*}{$\chi^{2}$} & \multirow[t]{3}{*}{$p$-Value } & \multirow[t]{3}{*}{ Odds (95\% Cl) } \\
\hline & & \multicolumn{2}{|r|}{ Absent } & \multicolumn{2}{|c|}{ Present } & & & \\
\hline & & Count & Percentage & Count & Percentage & & & \\
\hline \multirow[t]{2}{*}{ Size } & $>5$ & 19 & 65.5 & 10 & 34.5 & \multirow[t]{2}{*}{7.09} & \multirow[t]{2}{*}{$<0.01$} & - \\
\hline & $\leq 5$ & 16 & 100.0 & 0 & 0.0 & & & - \\
\hline \multirow[t]{2}{*}{ \% Necrosis } & High & 8 & 50.0 & 8 & 50.0 & \multirow[t]{2}{*}{11.08} & \multirow[t]{2}{*}{$<0.01$} & $13.50(2.37-76.80)$ \\
\hline & Low & 27 & 93.1 & 2 & 6.9 & & & 1 \\
\hline \multirow{3}{*}{$\begin{array}{l}\text { Shape/ } \\
\text { surface }\end{array}$} & Irregular and lobulated & 6 & 60.0 & 4 & 40.0 & \multirow[t]{3}{*}{4.02} & \multirow[t]{3}{*}{0.134} & - \\
\hline & Regular and lobulated & 15 & 75.0 & 5 & 25.0 & & & - \\
\hline & Regular and smooth & 14 & 93.3 & 1 & 6.7 & & & - \\
\hline \multirow{2}{*}{$\begin{array}{l}\text { Adjacent } \\
\text { organ } \\
\text { infiltration }\end{array}$} & Present & 6 & 46.2 & 7 & 53.8 & \multirow[t]{2}{*}{10.58} & \multirow[t]{2}{*}{$<0.001$} & $11.28(2.25-56.59)$ \\
\hline & Absent & 29 & 90.6 & 3 & 9.4 & & & 1 \\
\hline \multirow{2}{*}{$\begin{array}{l}\text { Mitotic } \\
\text { index }\end{array}$} & High & 10 & 52.6 & 9 & 47.4 & \multirow[t]{2}{*}{12.03} & \multirow[t]{2}{*}{$<0.01$} & $22.5(2.51-201.5)$ \\
\hline & Low & 25 & 96.2 & 1 & 3.8 & & & 1 \\
\hline
\end{tabular}

Abbreviations: $\mathrm{Cl}$, confidence interval; $\chi^{2}$, Chi square.

irregular contour, mild-to-moderate heterogenous pattern of enhancement, exophytic growth and necrosis $>30 \%$. The age and sex distribution of our cases as well as the CT morphological parameters were similar to what has been described in several previous studies. ${ }^{10-12}$

At present, risk stratification of GISTs is based on three parameters, namely, tumor size, organ of origin and mitotic rates. There have been numerous studies conducted on GISTs in the past, and more than eight established risk classification systems exist at present. Of these, the classification scores by $\mathrm{NIH}$ (Fletcher et al) and The Armed Forces Institute of Pathology (Miettinen and Lasota) are more universally accepted. ${ }^{13-15}$

There are conflicting reports in the available literature as far as the utility of imaging parameters (other than size and organ of origin) in risk assessment is concerned. In our study, we found significant association between percentage of necrosis and risk category. Percentage of necrosis showed fair agreement in predicting risk when cutoff was $50 \%$ and moderate agreement when the cutoff was fixed at 30\% $(p<0.01)$. None of the cases with $<30 \%$ necrosis were in the high-risk category. There was no significant association between percentage of necrosis and MI in our study. Several previous studies too have highlighted the importance of necrosis in risk stratification of GISTs. ${ }^{16}$ Liu et al conducted a study on 740 cases of GISTs, which showed that tumor necrosis was significantly associated with large tumor size, higher MI, tumor rupture and nuclear atypia. ${ }^{17}$ Gronchi et al have reported that necrosis and adjacent organ infiltration are associated with high risk and high cellularity. ${ }^{18}$

However, there are some studies like the one published by Ahmed et al which failed to show any correlation between radiological appearances such as necrosis, hemorrhage or cyst formation and malignant potential. ${ }^{19}$ This is probably due to the very small sample size of 24 cases and the short duration of follow-up.

We found that in our study, all tumors that were predominantly cystic ( $n=7$ ) belonged to the high-risk category ( - Fig. 8).
One of these cases was a female patient with history of NF1 who had a large duodenal cystic GIST, which was resistant to Imatinib and developed metastasis on follow-up (-Fig. 9A-G). It is well-documented that NF1 associated GISTs are mostly of the wild type and are resistant to Imatinib..$^{20,21}$ None of our remaining cases had syndromic association. Wang et al has stated that GISTs with predominant cystic change are seen in high-grade tumors, when they outgrow the blood supply due to aggressive growth and tumor necrosis. ${ }^{21}$

In our study, there was moderate association between GISTs with adjacent organ infiltration and high-risk category ( $p$-value of 0.01 ) as well as with high MI ( $p$-value of 0.003 ). Tumors with lobulated and irregular shape/surface characteristics also showed significant association with risk category ( $p$-value of 0.01) and with MI ( $p$-value of 0.04). Tateishi et al in their study concluded that the CT features that suggest a highgrade GIST and predict poor outcome include hepatic metastasis, irregular borders, adjacent organ invasion, and lesions larger than $11.1 \mathrm{~cm}^{22}$

When the size cutoff was $5 \mathrm{~cm}$, the accuracy to predict high risk was $82.2 \%$. It is well-documented that large size especially more than $10 \mathrm{~cm}$ is a strong predictor of metastasis. ${ }^{6,22}$ Among the 10 cases in the study that had metastasis at the time of presentation or on follow-up, $70 \%$ were more than $10 \mathrm{~cm}$ and all were more than $5 \mathrm{~cm}$. Extent of necrosis more than 30\% ( $p<0.01)$ and presence of adjacent organ infiltration ( $p$-value of 0.001 ) also showed significant association with development of metastasis. The most common site of metastasis was liver followed by peritoneum/omentum as documented in several previous studies. ${ }^{12}$

The role of a radiologist is limited in predicting risk stratification of GISTs; however, it is important to ascertain the size as well as the organ of origin accurately to guide the clinician and aid in risk stratification.

CT morphological parameters of significance in risk stratification as per our study include tumor necrosis, predominant cystic change, irregular and lobulated shape/surface characteristics and adjacent organ infiltration. 
Those parameters that predicted high MI were large size, irregular or lobulated shape/surface, endophytic growth pattern and adjacent organ infiltration.

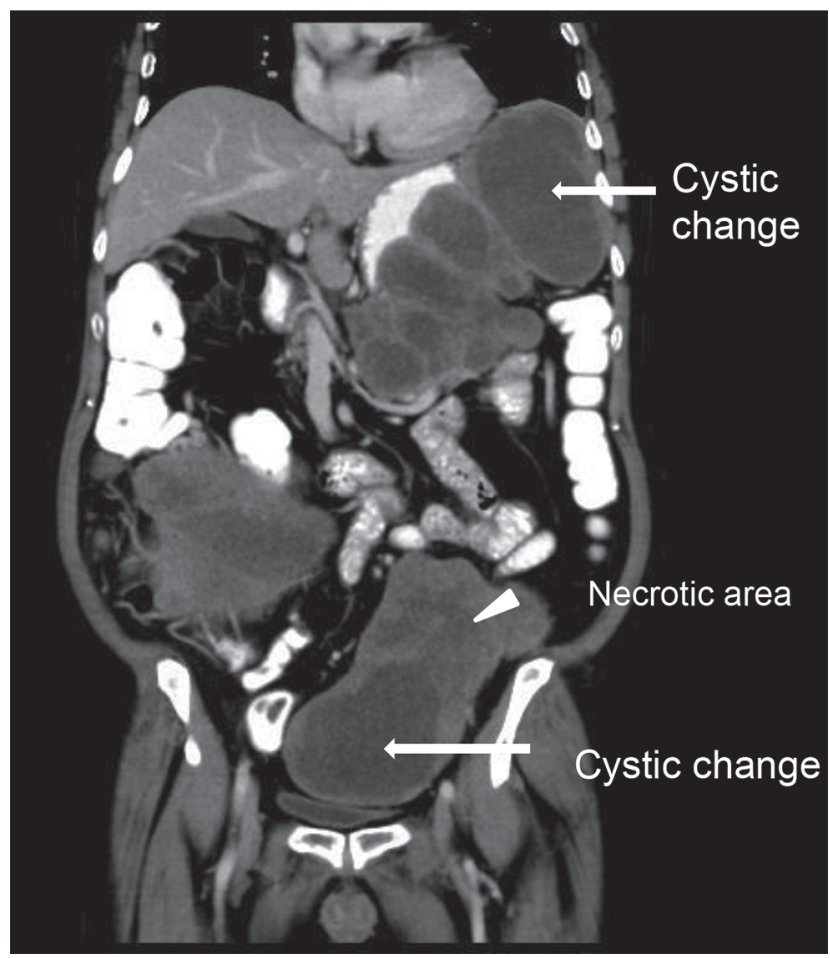

Fig. 8 Coronal contrast-enhanced CT image showing large exophytic gastric gastrointestinal stromal tumor (GIST) with predominant cystic change and multiple peritoneal deposits. Pelvic metastatic deposit shows necrotic area as well as cystic change. High risk. Mitotic index (MI): > 5/50 HPF. Size $19.1 \mathrm{~cm}$ (white arrows point to cystic change and white arrowhead points to necrotic area).
The parameters which were associated with development of metastasis were size $>5 \mathrm{~cm}$, necrosis $>30 \%$ and the presence of adjacent organ infiltration. None of the low-risk cases in our study, or those with $<30 \%$ necrosis, developed metastasis during follow-up. Only one (10\%) small bowel GIST with low MI had metastasis. Burkill et al in their study have reported the imaging features and metastatic pattern of GIST, but they have not correlated the imaging features with metastatic potential of GIST. ${ }^{23}$

\section{Pitfalls}

Our study had several limitations, the major factor being the relatively limited sample size. Among the 45 cases in the study, only 33 could be followed-up. We did not carry out separate assessment for gastric and nongastric GISTs. Only very few of our cases had PET CT evaluation and therefore we have not assessed its role in risk stratification. Due to the very small number of cases that had MRI evaluation in our study $(n=4)$, it was not feasible to ascertain the usefulness of this modality in GIST risk assessment or prediction of metastasis. We have not done a prospective study to verify the analysis.

\section{Conclusion}

The role of the radiologist is limited in predicting risk category of GISTs. However, if there are certain imaging morphological parameters which can predict risk category/ predisposition to develop metastasis, then it is of great value to the clinician in prognostication and treatment planning.

CT morphological parameters of significance in risk stratification, as per our study, include tumor necrosis $>30 \%$, predominant cystic change, irregular/lobulated shape/surface characteristics and presence of adjacent organ infiltration.
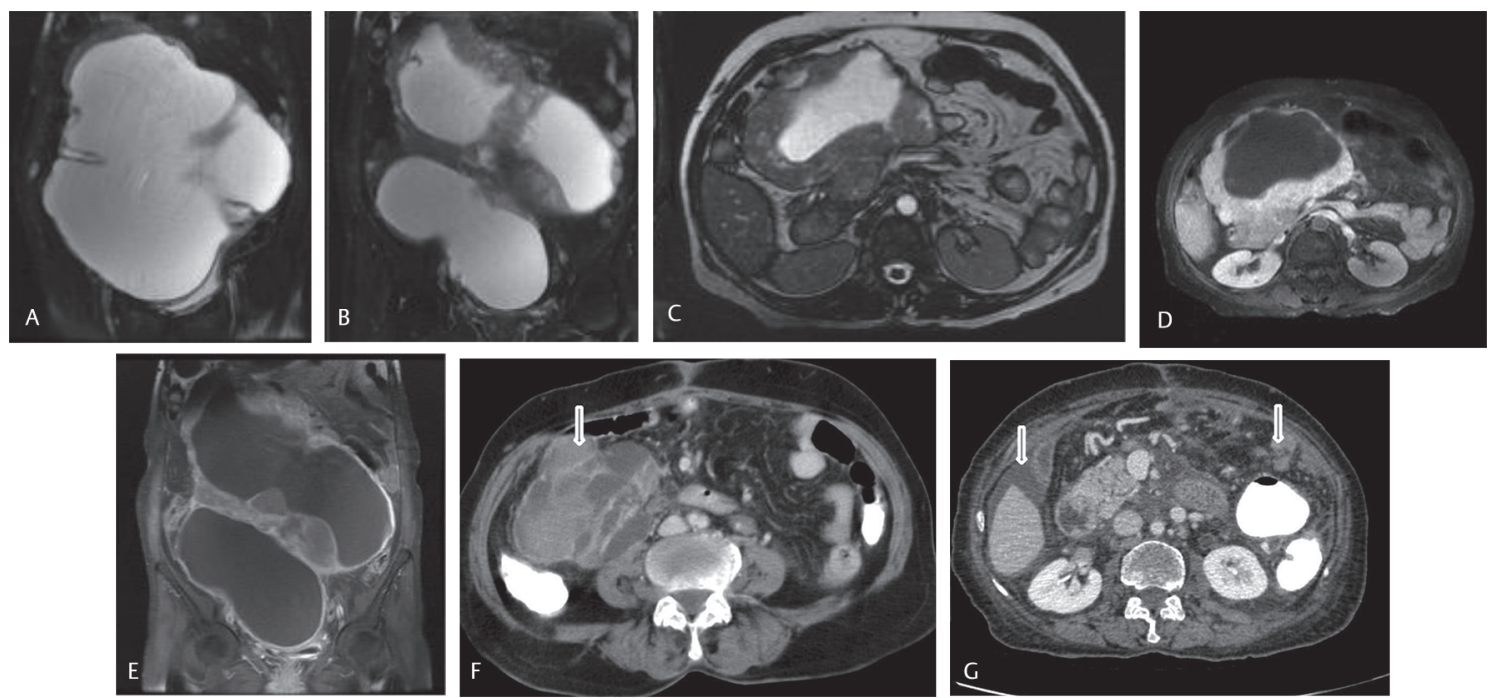

Fig. 9 Correlative MRI scan-Coronal T2WI images (A, B) of a known case of neurofibromatosis type I (NF1), showing large complex solid cystic lesion filling almost the whole abdomen. Axial FIESTA image (C) shows the superior end of the lesion indenting the 3rd segment of duodenum. Central cystic and peripheral solid components of lesion appreciable. Postcontrast axial (D) and coronal (E) T1W images show moderate to marked enhancement of the peripheral solid components. Postsurgical, follow-up contrast-enhanced CT axial images (F, G) after 6 months show recurrence in the right iliac fossa (solid cystic mass) along with ascites and multiple omental deposits (white arrows). High risk tumor with predominant cystic changes and size $>20 \mathrm{~cm}$ and mitotic index (MI) $>5 / 50 \mathrm{HPF}$ arising from duodenum with recurrence on follow-up. 


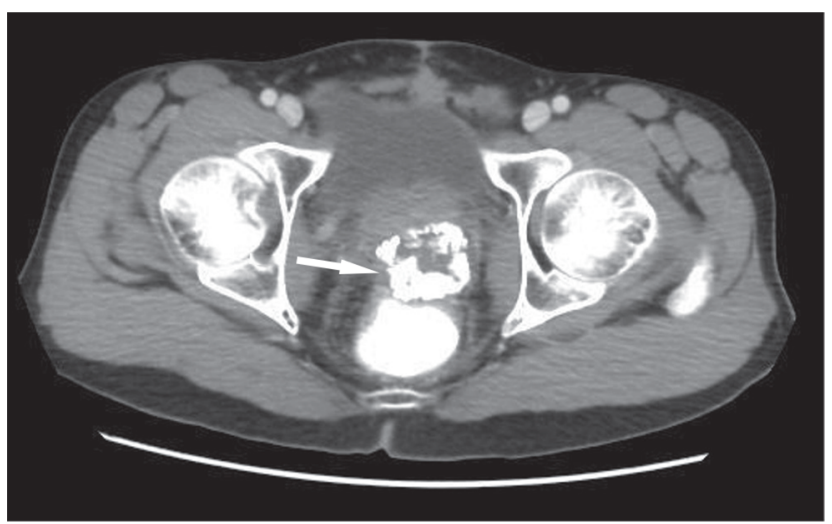

Fig. 10 Axial contrast-enhanced CT image showing partially calcified rectal gastrointestinal stromal tumor (white arrow). High risk. Mitotic index $(\mathrm{MI})>5 / 50 \mathrm{HPF}$. Size: $5 \mathrm{~cm}$.

Size $>5 \mathrm{~cm}$, along with irregular/lobulated contour, adjacent organ infiltration and endophytic growth pattern favored high MI.

The parameters which were associated with development of metastasis were size $>5 \mathrm{~cm}$, necrosis $>30 \%$ and the presence of adjacent organ infiltration. None of the patients with less than $30 \%$ necrosis developed metastasis in our study.

The radiologist also plays an important role in ascertaining the size of tumor and the organ of origin accurately to guide the clinician in risk calculation and subsequent prognostication.

Size and organ of origin are already known to have a significant role in risk stratification, and this was reinstated in our study. However, as these two parameters are already incorporated in risk classification, it is inappropriate to assess their association with risk. Nevertheless, the increased risk of extragastric GIST when compared with gastric GIST was reaffirmed. All four rectal GISTs in our study were in the high-risk category (-Fig. 10).

\section{Conflict of Interest}

None declared.

\section{Acknowledgments}

The authors would like to acknowledge Dr. Oommen P. Mathew, MSc, PGD (Com. Sc), PGD (Bio Stat), PhD (Demography). Research Investigator, Population Research Centre, University of Kerala. Dr. Shivaprakash B. Hiremath, Fellow in Neuroradiology, The Ottawa Hospital, University of Ottawa, Canada. Dr. Abraham Koshy, MD, DM (Gastroenterology) FAASLD, Director, Research \& Hepatology, VPS Lakeshore Hospital. Ms. Suja S, Research Scholar, Department of Electronics, CUSAT, Kochi.

\section{References}

1 Mazur MT, Clark HB. Gastric stromal tumors. Reappraisal of histogenesis. Am J Surg Pathol 1983;7(6):507-519

2 Liegl B, Hornick JL, Lazar AJ. Contemporary pathology of gastrointestinal stromal tumors. Hematol Oncol Clin North Am 2009;23(1):49-68, vii-viii

3 Miettinen M, Lasota J. Gastrointestinal stromal tumorsdefinition, clinical, histological, immunohistochemical, and molecular genetic features and differential diagnosis. Virchows Arch 2001;438(1):1-12
4 Caterino S, Lorenzon L, Petrucciani N, et al. Gastrointestinal stromal tumors: correlation between symptoms at presentation, tumor location and prognostic factors in 47 consecutive patients. World J Surg Oncol 2011;9:13

5 Sanchez-Hidalgo JM, Duran-Martinez M, Molero-Payan R, et al. Gastrointestinal stromal tumors: a multidisciplinary challenge. World J Gastroenterol 2018;24(18):1925-1941

6 O'Neill AC, Shinagare AB, Kurra V, et al. Assessment of metastatic risk of gastric GIST based on treatment-naïve CT features. Eur J Surg Oncol 2016;42(8):1222-1228

7 O'Regan KN, Shinagare AB, Saboo SS, Ramaiya NH, Jagannathan JP, Tirumani SH. Gastrointestinal stromal tumors (GIST): lesser known facts. Clin Imaging 2013;37(5):821-829

8 Joensuu H, Hohenberger P, Corless CL. Gastrointestinal stromal tumour. Lancet 2013;382(9896):973-983

9 Levy AD, Remotti HE, Thompson WM, Sobin LH, Miettinen M. Gastrointestinal stromal tumors: radiologic features with pathologic correlation. Radiographics 2003;23(2):283-304, 456, quiz 532

10 Kochhar R, Manoharan P, Leahy M, Taylor MB. Imaging in gastrointestinal stromal tumours: current status and future directions. Clin Radiol 2010;65(8):584-592

11 Catalano O, De Lutio di Castelguidone E, Nunziata A, De Rosa V, Siani A. Gastrointestinal stromal tumours: pictorial review. Radiol Med (Torino) 2005;110(5-6):484-491

12 Parab TM, DeRogatis MJ, Boaz AM, et al. Gastrointestinal stromal tumors: a comprehensive review. J Gastrointest Oncol 2019;10(1):144-154

13 Schmieder M, Henne-Bruns D, Mayer B, et al. Comparison of different risk classification systems in 558 patients with gastrointestinal stromal tumors after R0-resection. Front Pharmacol 2016;7:504

14 Agaimy A. Gastrointestinal stromal tumors (GIST) from risk stratification systems to the new TNM proposal: more questions than answers? A review emphasizing the need for a standardized GIST reporting. Int J Clin Exp Pathol 2010;3(5):461-471

15 Joensuu H. Risk stratification of patients diagnosed with gastrointestinal stromal tumor. Hum Pathol 2008;39(10):1411-1419

16 Koay MH, Goh YW, Iacopetta B, et al. Gastrointestinal stromal tumours (GISTs): a clinicopathological and molecular study of 66 cases. Pathology 2005;37(1):22-31

17 Liu X, Qiu H, Zhang P, et al; China Gastrointestinal Stromal Tumor Study Group (CN-GIST). Prognostic role of tumor necrosis in patients undergoing curative resection for gastric gastrointestinal stromal tumor: a multicenter analysis of 740 cases in China. Cancer Med 2017;6(12):2796-2803

18 Gronchi A. Risk stratification models and mutational analysis: keys to optimising adjuvant therapy in patients with gastrointestinal stromal tumour. Eur J Cancer 2013;49(4):884-892

19 Afifi AH, Eid M. Gastrointestinal stromal tumors (GISTs): diagnostic value of multi-detector computed tomography. The Egyptian Journal of Radiology and Nuclear Medicine 2012;43(2):139-146

20 Fujimi A, Nagamachi Y, Yamauchi N, et al. Gastrointestinal Stromal Tumor in a Patient with Neurofibromatosis Type 1 That Was Successfully Treated with Regorafenib. Intern Med 2019;58(13):1865-1870

21 Wang L, Liu L, Liu Z, Tian Y, Lin Z. Giant gastrointestinal stromal tumor with predominantly cystic changes: a case report and literature review. World J Surg Oncol 2017;15(1):220

22 Tateishi U, Hasegawa T, Satake M, Moriyama N. Gastrointestinal stromal tumor. Correlation of computed tomography findings with tumor grade and mortality. J Comput Assist Tomogr 2003;27(5):792-798

23 Burkill GJ, Badran M, Al-Muderis O, et al. Malignant gastrointestinal stromal tumor: distribution, imaging features, and pattern of metastatic spread. Radiology 2003;226(2):527-532 